\title{
Ampholytic Chitosan/Alginate Composite Nanofibrous Membranes with Super Anti-Crude Oil-Fouling Behavior and Multifunctional Oil/Water Separation Properties
}

Weikang Zhou, Yan Fang, Peiyuan Li, Liyu Yan, Xianmou Fan, Zhiguo Wang, Wen

\author{
Zhang, Haiqing Liu*
}

Fujian Provincial Key Laboratory of Polymer Materials, Fujian Normal University,

\section{Fuzhou 350007, China}

${ }^{*}$ Correspondence to: Haiqing Liu (E-mail: haiqingliu@ fjnu.edu.cn)

$$
\text { Yan Fang (E-mail:fangyan_YWJ@163.com) }
$$

Number of pages: 2

Number of figures: 3

Number of tables: 1
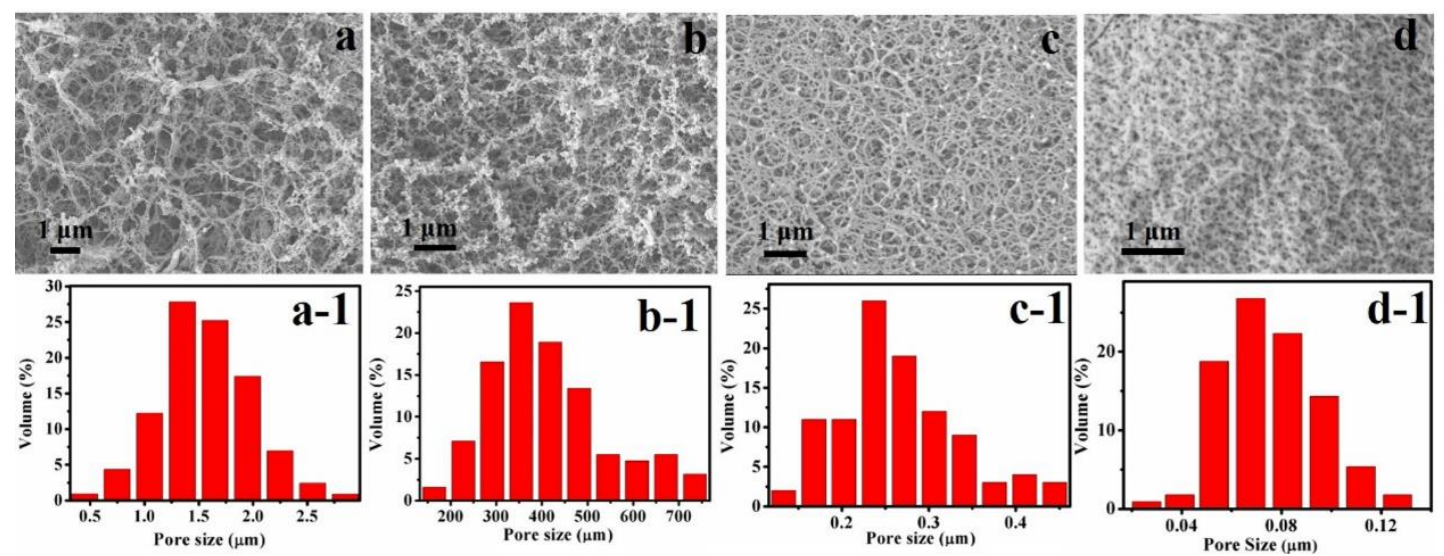

Figure S1. Surface images of CAM with various ECH content of (a) $1.5 \mathrm{wt} \%$, (b) $2 \mathrm{wt} \%$

(c) $2.5 \mathrm{wt} \%$, (d) $3 \mathrm{wt} \%$, and corresponding pore size distribution. 


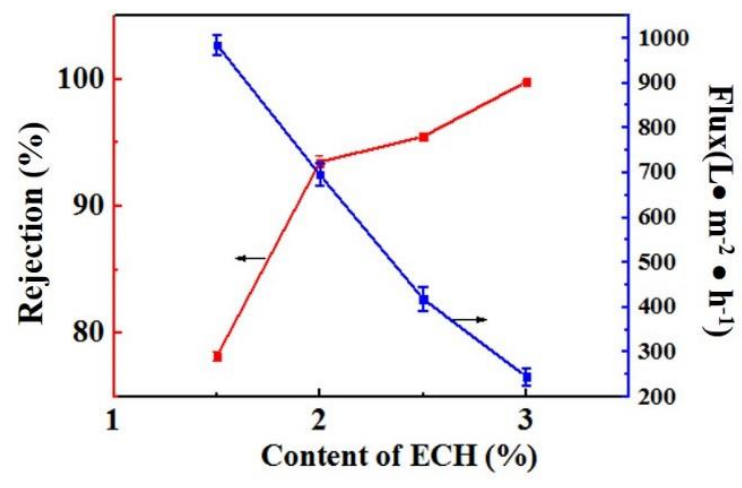

Figure S2. Crude oil rejection and water flux of CAM as a function of ECH content.

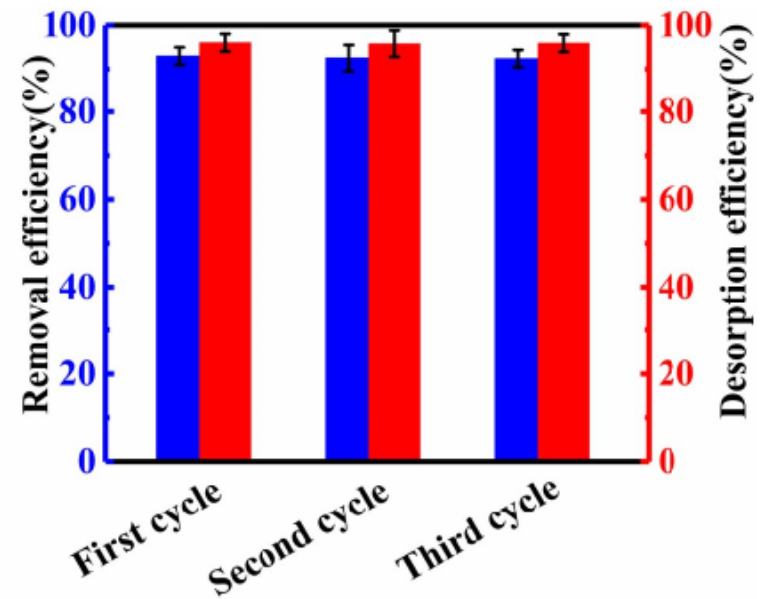

Figure S3. Adsorption-desorption cycle of $\mathrm{Zn}^{2+}$ ions by the CAM with ECH content of $2 \mathrm{wt} \%$.

Table S1 Zeta potential of CS, ALG and CAM

\begin{tabular}{cc}
\hline Sample & zeta potential $(\mathbf{m V})$ \\
\hline CS & $65.66 \pm 0.11$ \\
ALG & $-66.27 \pm 0.05$ \\
CAM & $-0.54 \pm 0.09$ \\
\hline
\end{tabular}

